$A \mathbb{N} A \mathbb{A} \mid$

Disponível em

http://www.anpad.org.br/rac

RAC, Curitiba, v. 15 , n. 5, art. 5,

pp. 877-896, Set./Out. 2011

$(\mathrm{cc})$ EY-NC

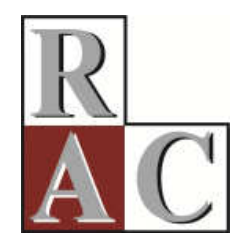

\title{
Uma Generalização Empírica sobre Comunicação Boca a Boca Usando Metanálise
}

Empirical Generalization Concerning Word-of-Mouth Marketing Using Meta-Analysis

Celso Augusto de Matos *

E-mail: celsoam@unisinos.br Universidade do Vale do Rio dos Sinos - PPG/UNISINOS

São Leopoldo, RS, Brasil.

* Endereço: Celso Augusto de Matos

UNISINOS, Centro 5, Sala 5A 403C, São Leopoldo/RS, CEP 93022-000.

Copyright (C) 2011 RAC. Todos os direitos, até mesmo de tradução, são reservados. É permitido citar parte de artigos sem autorização prévia, desde que seja identificada a fonte. 


\title{
Resumo
}

Com base em uma revisão da literatura nacional e estrangeira sobre comunicação boca a boca, propõe-se um modelo teórico no qual o constructo boca a boca (BAB) é considerado como foco; a satisfação e a lealdade são os antecedentes; e a valência de BAB (positivo, neutro ou negativo) é a moderadora. Esse modelo teórico é testado usando dados obtidos de uma metanálise. Após revisar 664 estudos, um subconjunto de 90 é submetido às análises, por conter as relações de interesse e trazer as estatísticas essenciais para a metanálise: a correlação entre os constructos e/ou estatísticas de diferenças de grupos. O subconjunto analisado produziu 119 amostras independentes e 36.673 respondentes. Os resultados mostraram uma associação significativa da satisfação e da lealdade com BAB. Além disso, a valência de BAB apresentou um efeito moderador significativo. Esse efeito moderador é proposto por esse trabalho como a seguinte generalização empírica: considerando a satisfação e a lealdade como variáveis associadas ao boca a boca, é a satisfação que possui relação mais forte com o boca a boca positivo, enquanto a lealdade é a que possui relação mais forte com o boca a boca negativo. Essa generalização pode ser simplificada pela expressão $\mathrm{SAT} \rightarrow \mathrm{BAB}(+), \mathrm{LEA} \rightarrow \mathrm{BAB}(-)$.

Palavras-chave: generalização empírica; metanálise; satisfação; lealdade; boca a boca.

\begin{abstract}
Based on a literature review of word-of-mouth communication, a theoretical framework is proposed and tested, in which word-of-mouth (WOM) is considered the main construct, satisfaction and loyalty are the antecedents and WOM valence (i.e., positive, negative and mixed) is the moderator. This theoretical model is tested using data obtained from a meta-analysis. After reviewing 664 studies, a total of 90 are submitted to the analyses, since they have the relationships of interest and the required statistics (i.e., correlations between constructs or statistics for group differences). The analyzed subset of studies produced 119 independent samples and 36,673 respondents. The results showed a significant association of satisfaction and loyalty with WOM. More specifically, WOM valence introduced a significant moderating effect. This moderating effect is proposed in this paper as the following empirical generalization: considering satisfaction and loyalty as correlates of WOM, satisfaction is the variable that has a stronger relationship with positive WOM, while loyalty is the one that is more closely associated with negative WOM, which might be expressed as SAT $\rightarrow$ WOM(+), LEA $\rightarrow$ WOM(-).
\end{abstract}

Key words: empirical generalization; meta-analysis; satisfaction; loyalty; word-of-mouth. 


\section{Introdução}

Sabe-se que o progresso de uma ciência se dá com o acúmulo de conhecimento dos estudos e o confronto das teorias com os dados empíricos. Nesse sentido, o Marketing, como outras ciências, busca interpretar o seu objeto de estudo dentro de arcabouços que permitam (a) orientar os objetivos da ciência, (b) oferecer um sistema de conceitos, (c) sintetizar o conhecimento por meio de generalizações empíricas e as inter-relações entre os conceitos, (d) fazer previsões e (e) indicar as lacunas do conhecimento (Hunt, 1983). As generalizações empíricas são de importância especial nesse sistema, visto que são os tijolos que constroem uma ciência (Bass \& Wind, 1995; Farley, Lehmann, \& Sawyer, 1995).

A maturidade do Marketing em alguns campos permite que se faça uma pausa e se questione o que foi aprendido até então e quais as questões mais importantes a serem aprofundadas em novas investigações. Um dos temas de marketing com esse perfil é o das comunicações boca a boca, chamado de word-of-mouth na literatura internacional. Esse tema recebeu atenção na Psicologia Social, a partir da importância das comunicações interpessoais nas escolhas dos indivíduos em diversos contextos, incluindo o de consumo (Katz \& Lazarsfeld, 1955). Nos estudos da disciplina de Comportamento do Consumidor, esse efeito das comunicações interpessoais também é relevante, principalmente quando o consumidor está na etapa de busca de informações e adquire um bem de alto envolvimento (Engel, Blackwell, \& Miniard, 1995). Na compra de serviços, o consumidor tende a se basear ainda mais nessas indicações de outras pessoas, pois os serviços são intangíveis, e assim, o consumidor, antes de comprar, utiliza referências como preço, evidências (aparências) do serviço, imagem da empresa e referências de pessoas próximas, como amigos e parentes, as chamadas comunicações boca a boca (Murray, 1991; Zeithaml, Berry, \& Parasuraman, 1993).

Nesse contexto, as comunicações boca a boca podem ser definidas como "comunicações informais direcionadas a outros consumidores sobre a posse, uso, ou características de produtos ou serviços e seus vendedores/fornecedores" (Westbrook, 1987, p. 261). Apesar da importância das comunicações interpessoais no marketing, são raros os estudos que consideram BAB como o constructo central e analisam os antecedentes e os moderadores, como destacado por Mazzarol, Sweeney e Soutar (2007, p. 1478). A satisfação tem recebido bastante atenção na literatura de marketing como antecedente principal do BAB (Mittal, Kumar, \& Tsiros, 1999; Oliver, 1980; Swan \& Oliver, 1989). A lealdade também foi proposta como uma antecedente do BAB (Dick \& Basu, 1994) e são diversos os estudos que correlacionam lealdade e boca a boca, mesmo que não apresentem uma ordem de influência (antecedente-consequente) entre esses dois constructos.

Embora haja grande volume de pesquisas relacionando esses três constructos (satisfação, lealdade e $\mathrm{BAB}$ ), há uma lacuna, em termos de uma revisão sistemática dessas relações e da análise dos fatores moderadores. A metanálise de Szymanski e Henard (2001) dá foco na satisfação e por isso considera somente esse antecedente de BAB, além de considerar somente BAB negativo. Dessa forma, o objetivo central deste trabalho é fazer uma revisão sistemática dos estudos nacionais e estrangeiros que relacionaram o $\mathrm{BAB}$ com satisfação e lealdade, a partir de um modelo teórico e do método de metanálise.

Além disso, há na literatura de $\mathrm{BAB}$ uma variação entre estudos que avaliam o $\mathrm{BAB}$ positivo, outros que avaliam o $\mathrm{BAB}$ negativo e ainda outros que adotam uma abordagem neutra, utilizando itens favoráveis e desfavoráveis para mensurar o constructo. Essa valência de BAB (positivo, negativo ou neutro), que encontra suporte nos estudos de Harrison-Walker (2001), pode também ajudar a explicar as relações de satisfação com BAB e lealdade com BAB. Portanto o segundo objetivo é testar esse fator (valência de $\mathrm{BAB}$ ) como moderador, conforme o modelo desenvolvido (ver Figura 1).

Por meio da metanálise, pode-se produzir uma revisão sistemática dos estudos prévios, considerando os erros de amostragem e de mensuração de cada estudo, analisar os antecedentes de $\mathrm{BAB}$ previstos no modelo teórico e identificar os efeitos da variável moderadora, contribuindo assim para a elaboração de generalizações empíricas sobre BAB (Farley et al., 1995). A metanálise é 
definida como "a análise estatística de um grande grupo de resultados de estudos individuais com o propósito de integrar os resultados" (Glass, 1976, p. 3). No Brasil, especificamente, a importância da metanálise já foi ressaltada por Pereira (2004) e estudos que aplicaram a técnica incluem Matos e Henrique (2006), Matos, Henrique e Rossi (2007), Matos e Rossi (2008) e Vieira (2008).

O artigo está estruturado da seguinte forma: na próxima seção é apresentado o modelo conceitual com as respectivas hipóteses. Em seguida, os procedimentos metodológicos conduzidos na metanálise são descritos. Faz-se, então, uma exposição dos dados e das análises centrais dentro dos objetivos deste trabalho. Essa análise é seguida pela discussão dos resultados e considerações finais, quando são apresentadas as contribuições, limitações e sugestões para novas pesquisas.

\section{Modelo Conceitual e Hipóteses}

Nesta seção, são apresentados o modelo teórico, a sua fundamentação na literatura e as relações esperadas para os seus constructos. A Figura 1 ilustra esse modelo teórico.

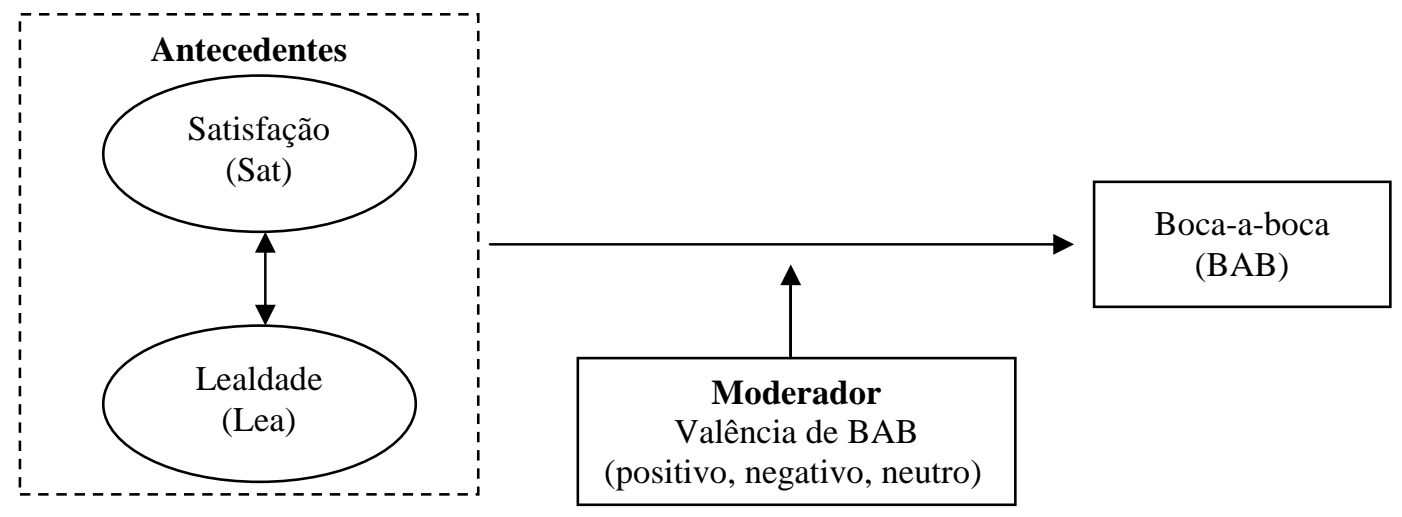

Figura 1. Modelo Teórico.

\section{A Relação entre Satisfação e Boca a Boca}

A satisfação é o sentimento do consumidor de que o consumo preenche, de modo favorável, alguma necessidade, desejo ou meta (Oliver, 1997, 1999). Nesta abordagem, a satisfação é definida como função do desempenho do produto em relação às expectativas (Oliver, 1980; Oliver \& Desarbo, 1988). Na literatura de marketing, o constructo satisfação é interpretado pela perspectiva da desconfirmação de expectativas, segundo a qual os consumidores são tidos como satisfeitos, quando os resultados superam as expectativas ou insatisfeitos se os resultados obtidos são inferiores às expectativas.

Em outra abordagem, considera-se que a satisfação não seja somente cognitiva, mas possua também um componente afetivo (Westbrook \& Oliver, 1991). Nessa linha, defende-se que as emoções sentidas durante o processo de consumo deixam traços afetivos na memória e estas são integradas posteriormente às avaliações de satisfação.

A satisfação é um dos conceitos centrais mais pesquisados em marketing (para uma revisão mais completa, ver Matos \& Henrique, 2006; Szymanski \& Henard, 2001) e uma pressuposição comum é que um consumidor satisfeito incorre em comportamentos favoráveis de compra, como repetição da compra, boca a boca positivo e propensão a maior tolerância em caso de problemas (Bearden \& Teel, 1983; Oliver, 1980; Ranaweera \& Prabhu, 2003; Richins, 1983). Anderson (1998) 
propõe que os consumidores nos extremos da avaliação de satisfação são os que têm maior chance de fazerem comunicações boca a boca, pois na situação de maior satisfação ativa o efeito do encantamento e na de menor satisfação, o arrependimento, em concordância com Oliver (1997) e Steiner, Schlemer e Pádua (2004).

Embora a maioria dos estudos que investigaram a relação entre satisfação e boca a boca encontraram suporte para um efeito significativo de satisfação no boca a boca (Heckman \& Guskey, 1998; Hennig-Thurau, Gwinner, \& Gremler, 2002; Mittal et al., 1999; Swan \& Oliver, 1989), há também aqueles estudos que não encontraram um efeito positivo e significativo de satisfação no boca a boca (Arnett, German, \& Hunt, 2003; Reynolds \& Beatty, 1999). Portanto esses resultados mistos também mostram que uma metanálise da relação entre satisfação e boca a boca pode contribuir para a literatura de marketing, principalmente porque a metanálise permite acumular os efeitos e controlar a influência de fatores externos (ex. erro de amostragem e confiabilidade das medidas), além de testar os efeitos de possíveis moderadores.

Dessa forma, propõe-se que:

H1: Há um efeito positivo e significativo da satisfação no boca a boca.

\section{A Relação entre Lealdade e Boca a Boca}

\section{No contexto de marketing, a lealdade pode ser definida como}

uma intenção de desempenhar um conjunto de comportamentos que sinalizem uma motivação de manter um relacionamento com a empresa em questão, incluindo alocação de mais recursos ao provedor de serviços, a recomendação por boca a boca positivo e a intenção de retorno (Sirdeshmukh, Singh, \& Sabol, 2002, p. 20).

Essa definição é derivada de Zeithaml, Berry e Parasuraman (1996).

Nota-se que essa definição considera o boca a boca positivo como parte do constructo lealdade. Essa prática tem sido adotada na literatura de marketing, como mostram os trabalhos de Zeithaml et al. (1996), Lam, Shankar, Erramilli e Murthy (2004), Bloemer, Ruyter e Wetzels (1999) e Jones e Sasser (1995). A pesquisa identificou que 46 estudos empregaram esta abordagem e não puderam ser incluídos nas análises, já que não apresentavam resultados específicos para o constructo BAB. Contudo cabe o questionamento: o constructo boca a boca deve ser considerado um constructo independente ou uma dimensão do constructo lealdade?

A fim de fornecer um argumento empírico para o questionamento acima, Söderlund (2006) fez o teste de dois modelos: (a) um em que os itens de intenções e de boca a boca foram considerados em conjunto num constructo nomeado como lealdade, modelado como consequente da satisfação e (b) outro em que dois constructos foram modelados como consequentes da satisfação: as intenções de compra e o boca a boca. Essa proposta foi testada em dois estudos e os resultados mostraram que as intenções e o boca a boca deveriam ser considerados constructos independentes, visto que quando foram comparados os ajustes dos dois modelos, o modelo com dois fatores separados (modelo b) produziu melhores índices de ajuste em relação ao modelo alternativo nos dois estudos, tanto no modelo de medida quanto no modelo estrutural.

Além disso, foi testado se o efeito da satisfação era diferente nos dois constructos (intenções e boca a boca). Foram encontradas diferenças nos dois estudos, pois em um deles a satisfação teve impacto mais forte no boca a boca e em outro nas intenções. Mesmo assim, essa abordagem de considerar intenções e boca a boca como dois constructos separados permite avaliar e comparar essas diferenças (Söderlund, 2006), o que não é possível quando todos os itens são agrupados num único constructo nomeado lealdade (a abordagem de coquetel de itens, não recomendada por Söderlund, 
2006). Outros estudos que também mediram intenções de recompra e boca a boca como constructos independentes incluem: Jones e Reynolds (2006), Gruen, Osmonbekov e Czaplewski (2006), Maxham e Netemeyer (2002a, 2002b), Maxham (2001), Blodgett, Granbois e Walters (1993).

A lealdade é proposta como antecedente de BAB com base na lógica de que clientes leais (a) fazem recomendações positivas da empresa aos indivíduos do seu grupo de referência (amigos e parentes); (b) têm maior motivação para processar novas informações sobre a empresa (ex. tomar conhecimento das inovações); e (c) possuem maior resistência a serem persuadidos por informação contrária às suas atitudes (Dick \& Basu, 1994, p. 107).

Porém esses autores reconheciam que na década de 1990 faltavam estudos empíricos que testassem essa relação lealdade $\rightarrow$ boca a boca. Ainda hoje, poucos são os estudos que testam empiricamente essa relação. Na revisão realizada nesta pesquisa, somente três referências foram encontradas (Carpenter \& Fairhurst, 2005; Gounaris \& Stathakopoulos, 2004; Reynolds \& Arnold, 2000). Estes estudos foram coerentes em apresentar um efeito positivo e significativo da lealdade sobre o BAB.

Mesmo assim, essa escassez de estudos, usando lealdade para prever BAB, não significa que esses constructos não estão associados (correlacionados) na literatura. De fato, há um número grande de estudos considerando lealdade e BAB como variáveis respostas, especialmente da satisfação (Arnett et al., 2003; Gremler \& Gwinner, 2000; Jones \& Reynolds, 2006; Macintosh, 2007; Maxham \& Netemeyer, 2002b, 2003; Price \& Arnould, 1999; Reynolds \& Beatty, 1999; Söderlund, 1998).

Portanto, se um dado estudo (a) propõe que a satisfação tem um efeito nesses constructos (lealdade e BAB), (b) mede esses constructos como variáveis independentes e (c) apresenta as correlações entre os pares de constructos, é possível incluir o dado estudo na metanálise e usar as correlações Sat-BAB e Lealdade-BAB. Assim, é possível incluir a relação entre lealdade e boca a boca na metanálise e avaliar os resultados médios dos estudos conduzidos, principalmente após a correção das diferenças entre os estudos.

Com base na proposição teórica de Dick e Basu (1994) e nos estudos empíricos que apoiaram a relação entre lealdade e $\mathrm{BAB}$, propõe-se que:

H2: Há um efeito positivo e significativo da lealdade no boca a boca.

\section{O Efeito Moderador da Valência do Boca a Boca}

As diferenças de efeitos entre os estudos podem ser devidas a diferenças de abordagens de BAB nas investigações. Por exemplo, na literatura de marketing, há estudos medindo BAB positivo, medindo $\mathrm{BAB}$ negativo e outros usando tanto itens positivos e negativos para medir $\mathrm{BAB}$ (BAB misto ou neutro).

Harrison-Walker (2001), em tentativa de desenvolver uma escala para medir BAB, definiu BAB teoricamente como constructo formado pelas facetas de frequência, número de contatos, detalhe da informação compartilhada e valência: quanto a comunicação é favorável ou desfavorável, também chamado de praise. Entretanto, no estudo empírico de Harrison-Walker (2001), somente duas dimensões emergiram no processo de purificação das medidas. A primeira dimensão foi nomeada como Atividade de BAB (WOM activity) e incluía aspectos de quão frequente acontece a comunicação de $\mathrm{BAB}$, para quantas pessoas o consumidor fez os comentários e a quantidade de informação fornecida pelo emissor. A segunda dimensão foi a Valência de BAB (WOM Praise), refletindo quanto era favorável/desfavorável as comunicações emitidas pelos respondentes. Essa distinção entre atividade de BAB e valência de BAB foi uma contribuição relevante de HarrisonWalker (2001) para a literatura sobre boca a boca. Harrison-Walker (2001) propõe que os pesquisadores que trabalharem com BAB devem considerar essas duas dimensões. 
Entretanto a maioria dos estudos na literatura de marketing geralmente mede o $\mathrm{BAB}$ como constructo unidimensional, que aborda a valência de $\mathrm{BAB}$ somente indiretamente por meio do uso de itens que refletem $\mathrm{BAB}$ favorável, desfavorável ou neutro. Por exemplo, um dado estudo que mede a propensão a $\mathrm{BAB}$ positivo usa um constructo unidimensional para $\mathrm{BAB}$ e não mede valência, mas considera essa dimensão, ao usar os itens de intenção de recomendações positivas. Se o estudo usa somente itens positivos, ele está medindo $\mathrm{BAB}$ positivo. Por outro lado, se o estudo usa somente itens negativos, está medindo BAB negativo. Em alguns casos, os estudos da literatura trabalham com uma posição neutra ou mista, combinando indicadores de recomendações positivas e negativas para o constructo $\mathrm{BAB}$.

No modelo teórico e nos procedimentos empíricos da meta-análise conduzida nesta pesquisa, foi seguida a abordagem de Harrison-Walker (2001), ou seja, de duas dimensões para BAB, ao estabelecer a atividade de $\mathrm{BAB}$ como variável resposta, e a valência de $\mathrm{BAB}$ como moderadora (ver Figura 1), isso porque o foco principal da literatura de $\mathrm{BAB}$ que foi revisada se baseia em aspectos da atividade de $\mathrm{BAB}$, incluindo a frequência, o número de pessoas contatadas e a quantidade de informações compartilhadas. A valência de $\mathrm{BAB}$ também é considerada, nesses estudos da literatura, mas indiretamente, ao mensurar BAB positivo, negativo ou neutro.

Ao adotar essa posição, reconhece-se no presente trabalho que não há somente um constructo $\mathrm{BAB}$ e que serão incluídos sob atividade de $\mathbf{B A B}$ os estudos que mediram a frequência de $\mathrm{BAB}, \mathrm{o}$ número relativo de pessoas contatadas e/ou a quantidade relativa de informação compartilhada, já que são essas as facetas da dimensão atividade de BAB apoiadas no estudo de Harrison-Walker (2001). A dimensão de valência de BAB é abordada como variável a ser testada como moderadora das relações Sat-BAB e Lealdade-BAB, ao separar os estudos pelas diferentes categorias da valência: positiva, negativa e neutra.

Assim, espera-se que quando o boca a boca assume a valência positiva, haja uma relação positiva dele com a satisfação e a lealdade, ao passo que, quando o boca a boca assume valência negativa, uma relação negativa é esperada. Mas, indo além, há razões para se hipotetizar que, para cada valência do boca a boca, os antecedentes satisfação e lealdade apresentem um efeito diferenciado sobre a atividade do boca a boca.

Por exemplo, Sweeney, Soutar e Mazzarol (2005) demonstraram empiricamente que o boca a boca positivo é mais cognitivo, mais cuidadosamente considerado e mais associado com comentários relacionados à qualidade do serviço recebido. De modo contrário, o boca a boca negativo é mais "passional", ou seja, baseado na emoção e passado adiante mais rapidamente. Dessa forma, o boca a boca negativo pode estar mais fortemente associado a intenções comportamentais, enquanto o boca a boca positivo pode ter um componente avaliativo (atitudinal) mais forte, sendo guiado por uma consideração racional mais cuidadosa. Esses diferentes papéis do boca a boca positivo e do boca a boca negativo também encontram suporte na Prospect Theory de Kahneman e Tversky (1979), segundo os quais perdas têm impacto maior que ganhos. Na mesma linha, Mittal, Ross e Baldasare (1998) mostraram que percepções negativas de desempenho possuem efeito maior na satisfação e nas intenções de recompra do que as percepções positivas de desempenho.

Considerando que a satisfação é usualmente abordada a partir de seus mecanismos cognitivos e que geralmente possui também um componente atitudinal (Oliver, 1980; Westbrook \& Oliver, 1991), especialmente quando os autores medem a satisfação acumulada, é razoável esperar que a satisfação (e não a lealdade) apresente relacionamento mais forte com o boca a boca positivo, já que esse tipo de boca a boca também depende de avaliações cognitivas e é cuidadosamente considerado antes de ser transmitido.

Por outro lado, em virtude da natureza emocional do boca a boca negativo (Schoefer \& Diamantopoulos, 2008; Sweeney, Soutar, \& Mazzarol, 2005) e sua maior associação com intenções e comportamento (isto é, o boca a boca negativo é transmitido mais rapidamente), pode-se esperar que a lealdade, ao invés da satisfação, esteja mais fortemente associada com o boca a boca negativo. Essa proposição é consistente com o argumento de Samson (2006) de que a lealdade a uma marca deve 
apresentar maior relação com o boca a boca negativo, quando comparado ao boca a boca positivo, porque o boca a boca é resultado direto da experiência do consumidor com a marca; experiências negativas com uma marca geralmente apresentam impacto maior sobre as intenções de recompra do que as experiências positivas. Com base nesta fundamentação, propõe-se que:

H3: A satisfação é mais fortemente associada ao boca a boca positivo, enquanto a lealdade é mais fortemente associada ao boca a boca negativo.

\section{Método}

\section{Processo de busca e codificação dos estudos}

Foi realizada uma revisão da literatura em diferentes bases científicas. Inicialmente, buscou-se por word-of-mouth e recommendation nos resumos e palavras-chave de cada um dos 28 journals de marketing que possuem fator de impacto, ou seja, que estão incluídos no Web of Science Journal Citation Reports (Recuperado em 07 de setembro, 2007, de http://apps.isiknowledge.com), usando Ebsco, Proquest e Elsevier Science Direct. Na sequência, foi realizada uma busca no Proquest, usando word-of-mouth em resumo e palavra-chave para todos os journals acadêmicos da base. O próximo passo foi realizar o mesmo procedimento anterior nas bases Ebsco, Emerald e Google Scholar, totalizando 536 estudos. A busca por dissertações e teses no Proquest resultou em 51 estudos. Finalizando a busca por artigos em língua estrangeira, foram enviados e-mails para 66 pesquisadores, cujos nomes apareciam com maior frequência na literatura, com o objetivo de obter artigos recentes que ainda não tinham sido publicados, bem como aqueles que não foram publicados no passado e ficaram na gaveta dos pesquisadores. Foram recebidos 17 artigos. Assim, um total de 591 estudos foi obtido na busca da literatura internacional; o artigo mais antigo era de 1955 e o mais atual de 2007. Todos os artigos identificados foram obtidos, seja por meio das bases citadas seja por pedido do artigo diretamente para os autores por e-mail.

A busca na literatura nacional se deu nas principais revistas brasileiras de marketing (RAC, RAE e RAUSP), nos Anais do Enanpad (1995 a 2007) e nos Anais do Encontro de Marketing (EMA), (2004 e 2006). Essas buscas resultaram em 73 artigos nacionais que haviam abordado o constructo boca a boca (medindo o constructo ou mencionando no texto o boca a boca ou a satisfação). Ressaltase que não houve limitação de período de tempo para a coleta nos periódicos internacionais nem nacionais. Somente na busca realizada nos Anais do Enanpad é que foi definido o período de 1995 a 2007, em função de acesso aos Anais pelo autor.

Portanto um total de 664 estudos $(591+73)$ foi obtido para a metanálise. Cada um desses estudos foi avaliado em termos das relações com BAB e seus antecedentes. Dois critérios foram usados para inclusão/exclusão: (a) estudos do tipo survey deveriam apresentar o coeficiente de correlação $(r)$ entre BAB e algum antecedente do modelo teórico (Figura 1); e (b) estudos que faziam comparações de grupos (ex. contraste de grupos por experimentos) deveriam apresentar as estatísticas relacionadas (ex. teste $t$, razão $F$ com um grau de liberdade no numerador) para a relação entre BAB e qualquer dos antecedentes. Em seguida, essas estatísticas seriam convertidas para o coeficiente $r$, usando as fórmulas comuns nos estudos de meta-análise (ver Lipsey \& Wilson, 2001, p. 198).

O coeficiente $r$ foi selecionado como a métrica para o effect size, porque ele é de fácil interpretação e uma medida que pode ser comparada entre estudos, já que é uma medida padronizada. Além disso, é a medida mais usualmente empregada nos estudos de metanálise na literatura de marketing (ex. Delvecchio, Henard, \& Freling, 2006; Eisend, 2004, 2006; Franke \& Park, 2006; Janiszewski, Noel, \& Sawyer, 2003; Palmatier, Dant, Grewal, \& Evans, 2006; Pan \& Zinkhan, 2006). Como foi incluído na base de dados tanto surveys como experimentos, foi possível combinar esses artigos ao usar $r$ como o effect size padrão. 
Usando esses procedimentos e finalizando as buscas em novembro de 2007, 91 estudos foram selecionados para fazer parte do banco de dados, o que resultou em uma base de dados com 309 effect sizes de 121 amostras independentes e um conjunto total de 46.080 respondentes. Na maioria dos casos (79 de 91 estudos), o coeficiente de correlação estava presente. Somente em 12 estudos o coeficiente $r$ foi derivado de um teste $t$ ou da estatística $F$ advindos de uma comparação de grupos.

Do total de 121 observações (amostras independentes), 60 (49\%) usaram o termo intenções de recompra (repurchase intentions), 22 (18\%) usaram lealdade (loyalty), 2 (2\%) mediram comportamento real e $37(31 \%)$ não mediram esse constructo. Dado que a maioria dos estudos mensurou intenções de lealdade, os constructos intenções de recompra e intenções de lealdade foram considerados como um constructo, sob o rótulo de lealdade.

\section{Procedimentos para a integração dos Effect Sizes}

Os dados foram digitados em uma planilha que continha os 91 estudos incluídos na metanálise. O objetivo era fazer a integração dos effect sizes de acordo com os princípios enunciados na literatura de metanálise (Lipsey \& Wilson, 2001; Wolf, 1986). Dado que a relação verdadeira entre duas variáveis é influenciada principalmente pelo erro de amostragem (o quão representativa é a amostra investigada) e o erro de mensuração (a confiabilidade das escalas), as correlações foram ponderadas inicialmente pela variância invertida e a seguir pela variância invertida corrigida pelo erro de mensuração.

Antes de aplicar a ponderação da variância (para corrigir o erro de amostragem) e o ajuste da confiabilidade (para corrigir o erro de mensuração), os valores originais de correlação foram convertidos para a estatística $Z$ de Fisher, seguindo a fórmula 1 . Após calcular os valores médios e intervalos de confiança para os valores de $Z$ de Fisher, eles foram convertidos de volta para $r$, usando a fórmula 2. Essas fórmulas estão disponíveis em Lipsey e Wilson (2001, pp. 63-64). Essa prática é usual em metanálises (ex. Grewal, Kavanoor, Fern, Costley, \& Barnes, 1997; Palmatier et al., 2006).

$$
\begin{aligned}
& \mathrm{ES}_{\mathrm{Z}_{\mathrm{r}}}=.5 \log _{\mathrm{e}}\left(\frac{1+\mathrm{r}}{1-\mathrm{r}}\right) \\
& r=\frac{e^{2 E S_{\mathrm{Zr}}}-1}{e^{2 E S_{\mathrm{Zr}}}+1}
\end{aligned}
$$

O ajuste para a confiabilidade foi realizado por meio da aplicação da ponderação pela confiabilidade diretamente à variância invertida, usando a fórmula 3. Nessa abordagem, o effect size é corrigido tanto pelo erro amostral quanto pelo erro de mensuração (Lipsey \& Wilson, 2001, p. 110).

$$
w^{\prime}=w \times r_{x x} \times r_{y y}
$$

Utilizando essa abordagem, a integração dos effect sizes é apresentada em três momentos: (a) correlações da forma como observadas nos estudos; (b) correlações corrigidas pelo erro amostral (ponderadas somente pela variância invertida); e (c) correlações corrigidas pelo erro amostral e pelo erro de mensuração (ponderadas tanto pela variância invertida quanto pela confiabilidade das escalas).

Um intervalo de confiança é apresentado para as médias dos effect sizes, e esse intervalo é significativo, quando não inclui o zero. Quando a média do effect size é significativa, uma estatística de fail safe $N$ é calculada a partir da fórmula 4. O fail safe estima o número de estudos não disponíveis e não significativos que seriam necessários para trazer a média do effect size para um nível não significativo (geralmente para um nível de 0,05, como é usual na literatura, Grewal et al., 1997). Essa estatística também é conhecida como file drawer problem (Rosenthal, 1979) e é um indicador da robustez dos resultados obtidos para o effect size médio calculado. 


$$
k_{0}=k\left(\frac{\overline{E S_{k}}}{\overline{E S_{c}}}-1\right)
$$

O teste $Q$ analisa a homogeneidade da distribuição dos effect sizes, a fim de avaliar se a variação entre os valores observados pode ser atribuída ao erro amostral ou a outros fatores intervenientes (moderadores). A estatística $Q$ é calculada pela fórmula 5 e segue uma distribuição qui-quadrado (Hedges \& Olkin, 1985). Se a hipótese nula de homogeneidade é rejeitada, a variação dos effect sizes de uma dada relação (ex. Sat-BAB) entre os diversos estudos pode ser atribuída a fatores que não o erro amostral sozinho, como variáveis moderadoras relacionadas às características dos estudos ou a diferentes abordagens adotadas nas investigações (Lipsey \& Wilson, 2001, p. 115).

$$
\mathrm{Q}=\left(\sum \mathrm{w}_{\mathrm{i}} \mathrm{ES}_{\mathrm{i}}^{2}-\frac{\left(\sum \mathrm{w}_{\mathrm{i}} \mathrm{ES}_{\mathrm{i}}\right)^{2}}{\sum \mathrm{w}_{\mathrm{i}}}\right)
$$

Quando os estudos mensuraram os constructos usando apenas um item (112 ou 33\% das 335 mensurações observadas), ou quando a confiabilidade não estava disponível nos estudos que usaram 2 ou mais itens para medir os constructos (19 de um total de 223 observações), essas confiabilidades foram estimadas usando o procedimento de Spearman-Brown, sugerido por Hunter e Schmidt (2004, p. 311). Esse método é usual em estudos prévios em marketing que fizeram metanálise (ex. Franke \& Park, 2006; Grewal et al., 1997). A vantagem desse procedimento de Spearman-Brown é que a confiabilidade estimada leva em consideração o número de itens que foi usado para medir a variável em questão, de modo que casos que não forneceram a confiabilidade, mas que mediram tal variável com números diferentes de indicadores, recebem diferentes confiabilidades estimadas.

\section{Resultados}

\section{Estatísticas descritivas}

Após revisar o tamanho da amostra de cada um dos 91 estudos do banco de dados, duas observações foram identificadas como outliers. Enquanto o tamanho de amostra dos demais estudos variava entre 40 e 1.405 , os dois outliers possuíam amostras de 6.201 e 3.206, respectivamente. Todas as análises apresentadas desse ponto em diante foram conduzidas após a exclusão desses outliers do banco de dados. Portanto, 90 estudos e 119 observações (amostras independentes) foram utilizados nas análises subsequentes.

$\mathrm{Na}$ Tabela 1 são apresentados os resultados para a integração dos effect sizes. Como se vê nessa tabela, no constructo satisfação, os 67 estudos produziram 92 amostras independentes (observações) no banco de dados. Já os 53 estudos de lealdade produziram 66 amostras independentes. 
Tabela 1

Estatísticas Descritivas para a Integração dos Effect Sizes

\begin{tabular}{|c|c|c|c|c|c|c|c|c|c|c|c|c|c|c|}
\hline Relação & $E^{\mathrm{a}}$ & $\underset{\mathrm{b}}{\mathrm{O}}$ & $\mathrm{N}^{\mathrm{c}}$ & Min. & Max. & $\begin{array}{l}\text { Média } \\
\text { simples } \\
r\end{array}$ & $\begin{array}{c}\text { Média } \\
\text { pond } \\
\text { pela } \\
\text { amostra } \\
r\end{array}$ & $\begin{array}{c}\text { Média } \\
\text { pond } \\
\text { pela } \\
\text { amostra } \\
\text { e } \\
\text { confiab. } \\
r\end{array}$ & Sig. & ICI & ICS & $Q$ & Sig & $\begin{array}{c}\text { Fail } \\
\text { Safe } \\
N\end{array}$ \\
\hline Sat-BAB & 67 & 92 & 26.531 & $-0,76$ & 0,95 & 0,47 & 0,48 & 0,50 & 0,000 & 0,49 & 0,51 & $5.251,5$ & 0,000 & 834 \\
\hline Lea-BAB & 53 & 66 & 21.072 & $-0,83$ & 0,93 & 0,38 & 0,37 & 0,39 & 0,000 & 0,37 & 0,40 & $5.637,9$ & 0,000 & 447 \\
\hline Sat-Lea & 42 & 54 & 15.427 & 0,12 & 0,98 & 0,70 & 0,71 & 0,72 & 0,000 & 0,71 & 0,73 & $2.037,1$ & 0,000 & 725 \\
\hline
\end{tabular}

Notas. (a) número de estudos; (b) número de observações; (c) amostra acumulada; Sat = satisfação; BAB = boca a boca; Lea $=$ lealdade; ICI = limite inferior do intervalo de confiança; $\mathrm{ICS}=$ limite superior do intervalo de confiança.

Com base em Cohen (1988), Lipsey e Wilson (2001) recomendam que effect sizes maiores que 0,40 podem ser considerados fortes e entre 0,10 e 0,25 médios. Essa interpretação é compartilhada por outros autores sobre o assunto (ex. Grissom \& Kim, 2005; Wolf, 1986). Assim, pode-se afirmar que o effect size médio de Sat-BAB é forte $(0,50)$ e o de Lea-BAB como médio $(0,39)$. Foi encontrada significância para os dois antecedentes de $\mathrm{BAB}$, apoiando as hipóteses $\mathrm{H} 1$ e H2.

Note-se aqui que as relações de satisfação e lealdade com $B A B$ incluem tanto estudos que avaliaram $\mathrm{BAB}$ negativo quanto $\mathrm{BAB}$ positivo. Por esse motivo, pode-se observar na Tabela 1 que a coluna de valores mínimos inclui correlações negativas para Sat-BAB e Lea-BAB (referindo-se aos casos que investigaram $\mathrm{BAB}$ negativo). Essa é uma possível razão para os efeitos de satisfação e lealdade em $\mathrm{BAB}$ ficarem nos níveis de 0,50 e 0,39. $\mathrm{Na}$ análise de moderadores, apresentada na próxima seção, esses efeitos serão calculados separadamente para os estudos que investigaram BAB positivo e aqueles que pesquisaram $\mathrm{BAB}$ negativo.

Ao examinar os intervalos de confiança (ICI e ICS) de cada relação apresentada na Tabela 1, pode-se notar que as relações Sat-BAB, Lea-BAB e Sat-Lea apresentam baixa dispersão em torno da média. A estatística de file drawer $N$ também foi alta nessas relações (maior que 447), indicando que um número grande de estudos com effect sizes não significativos seria necessário, a fim de reduzir o effect size médio calculado para um nível de não significância. Em outras palavras, esse resultado é uma evidência de que os effect sizes médios de Sat-BAB e Lea-BAB são improváveis de serem nulos, já que quanto maior o file drawer $N$, maior é a nossa confiança de que o effect size médio não é nulo. Por exemplo, para trazer o effect size médio da relação Sat-BAB para um nível médio de 0,05 (usado na pesquisa como base), seriam necessários 834 estudos com resultados nulos para serem incluídos nas análises.

Mas um conjunto de effect sizes bastante heterogêneos para as relações Sat-BAB e Lea-BAB, como indicado pela estatística $Q$, e também as altas amplitudes dos effect sizes nessas relações (ver coluna de mínimo e máximo) mostram que variáveis moderadoras (ex. a valência de BAB: positivo ou negativo) podem contribuir para explicar a variação desses effect sizes. Por esse motivo, as análises subsequentes avaliam o papel da valência de BAB como moderadora.

\section{Efeitos moderadores}

Do total de 119 observações, 74 estavam lidando com BAB positivo, 26 com BAB misto e 19 com BAB negativo. Conforme esperado, para os estudos de BAB negativo, havia uma relação negativa entre Sat e $\mathrm{BAB}$, indicando que maiores (menores) níveis de satisfação estavam associados a menores (maiores) níveis de BAB. Por outro lado, uma relação positiva foi encontrada entre os estudos que lidavam com $\mathrm{BAB}$ positivo. Também foi analisado o efeito médio para a situação de $\mathrm{BAB}$ 
misto, ou seja, quando $\mathrm{BAB}$ foi medido usando tanto itens favoráveis e desfavoráveis. Esses resultados segmentados por valência de BAB são apresentados na Tabela 2.

Tabela 2

Effect Sizes Médios Distribuídos por Valência de BAB

\begin{tabular}{|c|c|c|c|c|c|c|c|c|c|c|c|c|c|c|}
\hline Relação & $E^{\mathrm{a}}$ & $\mathrm{O}^{\mathrm{b}}$ & $\mathrm{N}^{\mathrm{c}}$ & Min. & Max. & $\begin{array}{c}\text { Média } \\
\text { simples } \\
r\end{array}$ & $\begin{array}{c}\text { Média } \\
\text { pond } \\
\text { pela } \\
\text { amostra } \\
r\end{array}$ & $\begin{array}{c}\text { Média } \\
\text { pond } \\
\text { pela } \\
\text { amostra } \\
\text { e confiab. } \\
r\end{array}$ & Sig. & ICI & ICS & $Q$ & Sig & $\begin{array}{c}\text { Fail } \\
\text { Safe } \\
N\end{array}$ \\
\hline Sat-BAB (+) & 44 & 58 & 17.708 & 0,14 & 0,95 & 0,64 & 0,62 & 0,63 & 0,000 & 0,62 & 0,64 & $1.666,7$ & 0,000 & 676 \\
\hline Sat-BAB (n) & 14 & 21 & 6.003 & $-0,36$ & 0,81 & 0,41 & 0,40 & 0,42 & 0,000 & 0,39 & 0,44 & 744,2 & 0,000 & 154 \\
\hline Sat-BAB (-) & 9 & 13 & 2.820 & $-0,76$ & $-0,22$ & $-0,48$ & $-0,48$ & $-0,50$ & 0,000 & $-0,54$ & $-0,47$ & 100,8 & 0,000 & 118 \\
\hline Lea-BAB (+) & 36 & 46 & 16.265 & $-0,01$ & 0,93 & 0,60 & 0,55 & 0,57 & 0,000 & 0,56 & 0,58 & $1.616,0$ & 0,000 & 480 \\
\hline Lea-BAB (n) & 8 & 9 & 1.673 & $-0,46$ & 0,77 & 0,33 & 0,25 & 0,30 & 0,000 & 0,24 & 0,35 & 222,4 & 0,000 & 44 \\
\hline Lea-BAB (-) & 9 & 11 & 3.134 & $-0,83$ & $-0,31$ & $-0,64$ & $-0,63$ & $-0,64$ & 0,000 & $-0,66$ & $-0,61$ & 125,78 & 0,000 & 129 \\
\hline
\end{tabular}

Nota. (a) número de estudos; (b) número de observações; (c) amostra acumulada; Sat = satisfação; BAB = boca-a-boca; Lea $=$ lealdade; $(\mathrm{n})$ = BAB neutro; $(-) \mathrm{BAB}$ negativo; $(+) \mathrm{BAB}$ positivo; $\mathrm{ICI}=$ limite inferior do intervalo de confiança; ICS = limite superior do intervalo de confiança.

Pode-se ver pela Tabela 2 que os estudos que trabalharam com o boca a boca positivo apresentaram uma relação positiva para satisfação-boca a boca, com uma média de $r=0,63$ ( $p<$ 0,000 ), e lealdade boca a boca (média de $r=0,57, p<0,000$ ). Uma comparação dessas médias mostrou uma diferença significativa $(z=6,26, p<0,000)$, indicando que o boca a boca positivo possui uma relação mais forte com a satisfação do que com a lealdade.

Por outro lado, conforme esperado, os estudos que mediram o boca a boca negativo apresentaram um efeito médio negativo em ambas as relações (satisfação boca a boca: $r=-0,50, p<$ 0,000; lealdade-boca a boca: $r=-0,64, p<0,000)$. A comparação dessas duas médias também revelou uma diferença significativa $(z=4,44, p<0,000)$. Esse resultado mostra que o boca a boca negativo possui uma associação mais forte com a lealdade do que com a satisfação.

Esses resultados apoiam os diferentes papéis da satisfação e da lealdade como antecedentes do boca a boca (H3), quando a valência do boca a boca assume a categoria positiva ou negativa.

\section{Discussão e Conclusões}

A metanálise apresentada neste artigo fornece uma revisão teórica e uma integração quantitativa de dois dos principais constructos que são associados com o BAB na literatura de marketing: satisfação e lealdade. Embora haja grande quantidade de estudos relacionando BAB com esses constructos, há falta de integração dessas relações na literatura de marketing, tanto nacional quanto internacional. Portanto a presente metanálise busca preencher essa lacuna, ao propor um modelo teórico que relaciona esses dois antecedentes com $\mathrm{BAB}$ e propõe a valência de $\mathrm{BAB}$ como moderadora. As contribuições da pesquisa são, portanto, derivadas do modelo conceitual proposto e da revisão meta-analítica.

Primeiro, o efeito positivo da satisfação e da lealdade sobre BAB mostrou que esses antecedentes possuem um efeito forte, mesmo quando se combinam estudos com diferentes 
abordagens de BAB. Esses resultados apoiam o papel relevante da satisfação e da lealdade na propensão do consumidor de emitir recomendações (positivas ou negativas) sobre um fornecedor, em concordância com a literatura (Brown, Barry, Dacin, \& Gunst, 2005; Gremler \& Gwinner, 2000; Jones \& Reynolds, 2006; Price \& Arnould, 1999; Söderlund, 2006).

Segundo, as análises para as relações Sat-BAB e Lea-BAB, segmentadas pela valência de BAB, ofereceram resultados interessantes. Foi encontrado que situações de $\mathrm{BAB}$ positivo produziam efeito médio positivo e mais forte para Sat-BAB $(0,63)$, quando comparada a Lea-BAB $(0,57)$. Porém situações de $\mathrm{BAB}$ negativo forneciam efeito médio negativo e mais forte para Lea-BAB $(-0,64)$, quando comparada a Sat-BAB (-0,50). Esses resultados sugerem que o BAB negativo possui uma associação mais forte com lealdade, em relação à satisfação. Mas o oposto é verdadeiro quando o BAB positivo é considerado: a sua associação é mais forte com a satisfação do que com a lealdade.

Embora a literatura tenha discutido se clientes com alta satisfação emitem mais ou menos BAB que clientes de baixa satisfação (Anderson, 1998), esta pesquisa traz novo ponto para discussão: em situação de alta satisfação, a satisfação parece ser um preditor mais forte de $\mathrm{BAB}$, mas em situação de baixa satisfação (isto é, clientes emitindo $\mathrm{BAB}$ negativo), a lealdade se torna o preditor mais forte de BAB. Este efeito é nomeado pelo autor como efeito $\mathbf{S a t} \rightarrow \mathbf{B A B}(+), \mathbf{L e a} \rightarrow \mathbf{B A B}(-)$ e é proposto como uma generalização empírica, definida como: considerando a satisfação e a lealdade como variáveis associadas ao boca a boca, é a satisfação que possui associação mais forte com o boca a boca positivo, enquanto é a lealdade (ou quebra da mesma) que possui associação mais forte com o boca a boca negativo.

Esse efeito pode ser explicado pelas diferenças entre atitudes e intenções comportamentais. Isto é, dado que (a) o boca a boca positivo é mais fortemente relacionado a avaliações cognitivas e é cuidadosamente considerado antes de ser emitido (Sweeney et al., 2005) e (b) a satisfação é geralmente interpretada por seus mecanismos cognitivos e possui um componente atitudinal (Oliver, 1980; Westbrook \& Oliver, 1991), pode-se esperar mesmo que a satisfação tenha um relacionamento mais forte para o boca a boca com valência positiva.

Por outro lado, dado que o boca a boca com valência negativa é mais baseado na emoção, sendo transmitido com maior rapidez e tendo, portanto, maior associação com um componente comportamental ou de intenções de comportamento (Schoefer \& Diamantopoulos, 2008; Sweeney et al., 2005), passa a ter sentido o resultado de que a lealdade (ou quebra da mesma) é mais fortemente associada ao boca a boca com valência negativa. Além disso, considerando-se que as perdas costumam ter maior peso para os indivíduos do que os ganhos (Prospect Theory, Kahneman \& Tversky, 1979), é mais provável que situações de boca a boca negativo tenham maior associação com o comportamento do consumidor (ex. quebra da lealdade).

Esses resultados também são consistentes com a noção de que o boca a boca negativo é usualmente um comportamento de clientes que trocam de provedor (isto é, quebram a lealdade) e a seguir emitem avaliações e recomendações negativas do antigo provedor como forma de reduzir a dissonância cognitiva experimentada após a decisão de troca (Wangenheim, 2005).

O suporte dessa hipótese de que a satisfação e a lealdade desempenham diferentes papéis na relação com o boca a boca, quando são consideradas as modalidades de boca a boca positivo e boca a boca negativo é consistente também com um estudo de Martin, O’Neill, Hubbard e Palmer (2008). Esses autores demonstram que uma medida de satisfação mais baseada em emoções, quando comparada a uma abordagem mais tradicional, baseada em medidas cognitivas, é uma preditora mais forte das intenções comportamentais futuras.

É mais provável, segundo os estudos, que os consumidores tendam ao boca a boca, quando eles passam por experiências emocionais significativas (Dick \& Basu, 1994; Söderlund \& Rosengren, 2007). A meta-análise apresentada neste artigo confirma essa noção de que um tipo de boca a boca mais baseado em emoções apresenta uma associação mais forte com as intenções comportamentais dos consumidores. 
Portanto a associação mais forte da satisfação com o boca a boca positivo e da lealdade (quebra da lealdade) com o boca a boca negativo pode ser considerada uma proposição nova dentro da teoria das comunicações boca a boca e, como é um padrão obtido com base na revisão de estudos empíricos, pode ser considerada como generalização empírica. Novos estudos podem e devem submeter essa generalização a novos testes e aprofundar o conhecimento nesta área.

De fato, esta metanálise pode motivar os pesquisadores em marketing a conduzirem futuras pesquisas em um conjunto de questões. Primeiro, investigações futuras relacionando o boca a boca com satisfação e lealdade podem ser aprofundadas, ao incluir emoções específicas (ex. raiva, arrependimento, frustração, desapontamento etc.) e avaliar a diferença que tais medidas possuem para explicar o comportamento e a intenção do consumidor de se comprometer em comunicação boca a boca, especialmente o boca a boca negativo, que foi visto como mais emocional e com um componente comportamental mais forte. Pesquisas nesse assunto poderão também investigar com mais detalhes essas emoções e comparar o papel delas em situações de boca a boca positivo e boca a boca negativo.

Segundo, já que a satisfação apresentou uma associação forte e significativa com BAB, e a satisfação reflete um afeto positivo em relação ao provedor/fornecedor, poder-se-á aprofundar em novas investigações os mecanismos afetivos por trás das relações entre satisfação e BAB. Além disso, a discussão dos fatores cognitivos e afetivos que influenciam a satisfação no contexto de recuperação de serviços (Andreassen, 2000) poderia ser expandida para a relação entre satisfação e BAB.

Outro aspecto relevante para os pesquisadores avaliarem é se os efeitos apresentados como significativos anteriormente, tanto em termos de antecedentes como de moderadores, são também relevantes, quando se considera o boca a boca eletrônico (ex. clientes fazendo recomendações pela Internet) e a maior interatividade desse contexto. Embora a literatura já tenha abordado o conceito de boca a boca eletrônico (ex. Andrade, Mazzon, \& Katz, 2006; Gruen, Osmonbekov, \& Czaplewski, 2006; Hennig-Thurau, Gwinner, Walsh, \& Gremler, 2004), mais pesquisas são necessárias para investigar no contexto de boca a boca eletrônico os pontos específicos levantados neste artigo.

Por fim, algumas das limitações que são comuns a revisões do tipo metanálise também estiveram presentes nesta pesquisa. Por exemplo, vários estudos não puderam ser incluídos, por razões como a falta de informações sobre as correlações das variáveis, mesmo quando estudos se baseavam nesta matriz para uma análise de regressão (somente os betas eram apresentados). Outra situação impeditiva era a mensuração de $\mathrm{BAB}$ juntamente com outros itens dentro de um constructo mais amplo de lealdade ou intenções comportamentais, a chamada abordagem de coquetel (Söderlund, 2006).

Além disso, as análises de moderadores se restringiram à variável que media a valência do boca a boca (positivo, negativo ou neutro). Isso foi uma limitação decorrente da evolução da pesquisa, pois era a variável disponível no estágio atual da pesquisa. Outras variáveis moderadoras que poderão ser incluídas nas futuras análises são: (a) o tipo de boca a boca (se recebido ou emitido pelo consumidor); (b) a incidência (se é uma intenção de boca a boca ou um comportamento relatado); (c) as características dos estudos em termos de pesquisa (survey ou experimento), design (cross-sectional ou longitudinal), respondentes (estudantes ou não) e contexto (serviços ou não).

Em resumo, esta pesquisa demonstra, com base em uma revisão metanalítica, que a influência da satisfação é mais forte para o boca a boca positivo, ao passo que a lealdade, ou quebra, tem maior associação com o boca a boca negativo. Espera-se que esses resultados contribuam com novos insights para os pesquisadores de marketing continuarem avançando a teoria sobre as comunicações boca a boca.

Artigo recebido em 27.04.2010. Aprovado em 05.05.2011. 


\section{Referências}

Anderson, E. W. (1998). Customer satisfaction and word-of-mouth. Journal of Service Research, 1(1), 5-17. doi: $10.1177 / 109467059800100102$

Andrade, J., Mazzon, J. A., \& Katz, S. (2006, maio). Boca-a-boca eletrônico: explorando e integrando conceitos de marketing viral, buzz marketing e word-of-mouse. Anais do Encontro de Marketing da ANPAD, Rio de Janeiro, RJ, Brasil, 2.

Andreassen, T. W. (2000). Antecedents to satisfaction with service recovery. European Journal of Marketing, 34(1-2), 156-175. doi: 10.1108/03090560010306269

Arnett, D. B., German, S. D., \& Hunt, S. D. (2003). The identity salience model of relationship marketing success: the case of nonprofit marketing. Journal of Marketing, 67(2), 89-105. doi: 10.1509/jmkg.67.2.89.18614

Bass, F. M., \& Wind, J. (1995). Introduction to the special issue: empirical generalization in marketing. Marketing Science, 14(3), G1-G5. doi: 10.1287/mksc.14.3.G1

Bearden, W. O., \& Teel, J. E. (1983). Selected determinants of consumer satisfaction and complaint reports. Journal of Marketing Research, 20(1), 21-28. doi: 10.2307/3151408

Blodgett, J. G., Granbois, D. H., \& Walters, R. G. (1993). The effects of perceived justice on complainants' negative word-of-mouth behavior and repatronage intentions. Journal of Retailing, 69(4), 399-428. doi: 10.1016/0022-4359(93)90015-B

Bloemer, J., Ruyter, K. de, \& Wetzels, M. (1999). Linking perceived service quality and service loyalty: a multi-dimensional perspective. European Journal of Marketing, 33(11-12), 10821106. doi: $10.1108 / 03090569910292285$

Brown, T. J., Barry, T. E., Dacin, P. A., \& Gunst, R. F. (2005). Spreading the word: investigating antecedents of consumers' positive word-of-mouth intentions and behaviors in a retailing context. Journal of the Academy of Marketing Science, 33(2), 123-138. doi: $10.1177 / 0092070304268417$

Carpenter, J. M., \& Fairhurst, A. (2005). Consumer shopping value, satisfaction, and loyalty for retail apparel brands. Journal of Fashion Marketing and Management, 9(3), 256-269. doi: $10.1108 / 13612020510610408$

Cohen, J. (1988). Statistical power analysis for the behavioral sciences (2nd ed.). Hillsdale, NJ: Lawrence Erlbaum Associates.

Delvecchio, D., Henard, D. H., \& Freling, T. H. (2006). The effect of sales promotion on postpromotion brand preference: a meta-analysis. Journal of Retailing, 82(3), 203-213. doi: 10.1016/j.jretai.2005.10.001

Dick, A. S., \& Basu, K. (1994). Customer loyalty: toward an integrated conceptual framework. Journal of the Academy of Marketing Science, 22(2), 99-113. doi: 10.1177/0092070394222001

Eisend, M. (2004). Is it still worth to be credible? A meta-analysis of temporal patterns of source credibility effects in marketing. Advances in Consumer Research, 31(1), 352-357.

Eisend, M. (2006). Two-sided advertising: a meta-analysis. International Journal of Research in Marketing, 23(2), 187-198. doi: 10.1016/j.ijresmar.2005.11.001 
Engel, J. F., Blackwell, R. D., \& Miniard, P. W. (1995). Consumer behavior (8th ed.). Florida: The Dryden Press.

Farley, J. U., Lehmann, D. R., \& Sawyer, A. (1995). Empirical marketing generalization using metaanalysis. Marketing Science, 14(3), G36-G46. doi: 10.1287/mksc.14.3.G36

Franke, G. R., \& Park, J. E. (2006). Salesperson adaptive selling behavior and customer orientation: a meta-analysis. Journal of Marketing Research, 43(4), 693-702. doi: 10.1509/jmkr.43.4.693

Glass, G. V. (1976). Primary, secondary, and meta-analysis of research. Educational Researcher, 5(10), 3-8. doi: 10.3102/0013189X005010003

Gounaris, S., \& Stathakopoulos, V. (2004). Antecedents and consequences of brand loyalty: an empirical study. Journal of Brand Management, 11(4), 283-306. doi: 10.1057/palgrave.bm.2540174

Gremler, D. D., \& Gwinner, K. P. (2000). Customer-employee rapport in service relationships. Journal of Service Research, 3(1), 82-104. doi: 10.1177/109467050031006

Grewal, D., Kavanoor, S., Fern, E. F., Costley, C., \& Barnes, J. (1997). Comparative versus noncomparative advertising: a meta-analysis. Journal of Marketing, 61(4), 1-15. doi: $10.2307 / 1252083$

Grissom R. J., \& Kim, J. J. (2005). Effect sizes for research: a broad practical approach. Mahwah, NJ: Lawrence Erlbaum Associates.

Gruen, T. W., Osmonbekov, T., \& Czaplewski, A. J. (2006). eWOM: the impact of customer-tocustomer online know-how exchange on customer value and loyalty. Journal of Business Research, 59(4), 449-456. doi: 10.1016/j.jbusres.2005.10.004

Harrison-Walker, L. J. (2001). The measurement of word-of-mouth communication and an investigation of service quality and customer commitment as potential antecedents. Journal of Service Research, 4(1), 60-75. doi: 10.1177/109467050141006

Heckman, R., \& Guskey, A. (1998). The relationship between alumni and university: toward a theory of discretionary collaborative behavior. Journal of Marketing Theory and Practice, 6(2), 97112.

Hedges, L. V., \& Olkin, I. (1985). Statistical methods for meta-analysis. Orlando, FL: Academic Press.

Hennig-Thurau, T., Gwinner, K. P., \& Gremler, D. D. (2002). Understanding relationship marketing outcomes: an integration of relational benefits and relationship quality. Journal of Service Research, 4(3), 230-247. doi: 10.1177/1094670502004003006

Hennig-Thurau, T., Gwinner, K. P., Walsh, G., \& Gremler, D. D. (2004). Electronic word-of-mouth via consumer-opinion platforms: what motivates consumers to articulate themselves on the internet? Journal of Interactive Marketing, 18(1), 38-52. doi: 10.1002/dir.10073

Hunt, S. D. (1983). Marketing theory: the philosophy of marketing science. Homewood, IL: Richard D. Irwin.

Hunter, J. E., \& Schmidt, F. L. (2004). Methods of meta-analysis: correcting error and bias in research findings (2nd ed.). Beverly Hills, CA: Sage. 
Janiszewski, C., Noel, H., \& Sawyer, A. G. (2003). A meta-analysis of the spacing effect in verbal learning: implications for research on advertising repetition and consumer memory. Journal of Consumer Researcher, 30(1), 138-149. doi: 10.1086/374692

Jones, M. A., \& Reynolds, K. E. (2006). The role of retailer interest on shopping behavior. Journal of Retailing, 82(2), 115-126. doi: 10.1016/j.jretai.2005.05.001

Jones, T. O., \& Sasser, W. E., Jr. (1995). Why satisfied customers defect. Harvard Business Review, 73(6), 88-91.

Kahneman, D., \& Tversky, A. (1979). Prospect theory: an analysis of decision under risk. Econometrica, 47(2), 263-291. doi: 10.2307/1914185

Katz, E., \& Lazarsfeld, P. F. (1955). Personal influence. New York: Free Press.

Lam, S. Y., Shankar, V., Erramilli, M. K., \& Murthy, B. (2004). Customer value, satisfaction, loyalty, and switching costs: an illustration from a business-to-business service context. Journal Academy of Marketing Science, 32(3), 293-311. doi: 10.1177/0092070304263330

Lipsey, M. W., \& Wilson, D. B. (2001). Practical meta-analysis. Beverly Hills, CA: Sage.

Macintosh, G. (2007). Customer orientation, relationship quality, and relational benefits to the firm. Journal of Services Marketing, 21(3), 150-159. doi: 10.1108/08876040710746516

Martin, D., O'Neill, M., Hubbard, S., \& Palmer, A. (2008). The role of emotion in explaining consumer satisfaction and future behavioural intention. Journal of Services Marketing, 22(3), 224-236. doi: 10.1108/08876040810871183

Matos, C. A. de, \& Henrique, J. L. (2006, setembro). Balanço do conhecimento em marketing: uma meta-análise dos resultados empíricos dos antecedentes e conseqüentes da satisfação e lealdade. Anais do Encontro Nacional da Associação Nacional de Pós-Graduação e Pesquisa em Administração, Salvador, BA, Brasil, 30.

Matos, C. A. de, Henrique, J. L., \& Rossi, C. A. V. (2007). Service recovery paradox: a meta-analysis. Journal of Service Research, 10(1), 60-77. doi: 10.1177/1094670507303012

Matos, C. A. de, \& Rossi, C. A. V. (2008). Word-of-mouth communications in marketing: a metaanalytic review of the antecedents and moderators. Journal of the Academy of Marketing Service, 36(4), 578-596. doi: 10.1007/s11747-008-0121-1

Maxham, J. G., III (2001). Service recovery's influence on consumer satisfaction, positive word-ofmouth, and purchase intentions. Journal of Business Research, 54(1), 11-24. doi: 10.1016/S0148-2963(00)00114-4

Maxham, J. G., III, \& Netemeyer, R. G. (2002a). A longitudinal study of complaining customers' evaluations of multiple service failures and recovery efforts. Journal of Marketing, 66(4), 57-71. doi: 10.1509/jmkg.66.4.57.18512

Maxham, J. G., III, \& Netemeyer, R. G. (2002b). Modeling customer perceptions of complaint handling over time: the effects of perceived justice on satisfaction and intent. Journal of Retailing, 78(4), 239-252. doi: 10.1016/S0022-4359(02)00100-8

Maxham, J. G., III, \& Netemeyer, R. G. (2003). Firms reap what they sow: the effects of shared values and perceived organizational justice on customers' evaluations of complaint handling. Journal of Marketing, 67(1), 46-62. doi: 10.1509/jmkg.67.1.46.18591 
Mazzarol, T., Sweeney, J. C., \& Soutar, G. N. (2007). Conceptualizing word-of-mouth activity, triggers and conditions: an exploratory study. European Journal of Marketing, 41(11-12), 14751494. doi: $10.1108 / 03090560710821260$

Mittal, V., Kumar, P., \& Tsiros, M. (1999). Attribute-level performance, satisfaction, and behavioral intentions over time: a consumption-system approach. Journal of Marketing, 63(2), 88-101. doi: $10.2307 / 1251947$

Mittal, V., Ross, W. T., \& Baldasare, P. M. (1998). The asymmetric impact of negative and positive attribute-level performance on overall satisfaction and repurchase intentions. Journal of Marketing, 62(1), 33-47. doi: 10.2307/1251801

Murray, K. B. (1991). A test of services marketing theory: consumer information acquisition activities. Journal of Marketing, 55(1), 10-25. doi: 10.2307/1252200

Oliver, R. L. (1980). A cognitive model of the antecedents and consequences of satisfaction decisions. Journal of Marketing Research, 17(4), 460-469. doi: 10.2307/3150499

Oliver, R. L. (1997). Satisfaction: a behavioral perspective on the consumer. New York: The McGraw-Hill Companies.

Oliver, R. L. (1999). Whence consumer loyalty? [Edição Especial] Journal of Marketing, 63, 33-44. doi:10.2307/1252099

Oliver, R. L., \& Desarbo, W. S. (1988). Response determinants in satisfaction judgments. Journal of Consumer Research, 14(4), 495-507. doi: 10.1086/209131

Palmatier, R. W., Dant, R. P., Grewal, D., \& Evans, K. R. (2006). Factors influencing the effectiveness of relationship marketing: a meta-analysis. Journal of Marketing, 70(4), 136-153. doi: $10.1509 / j m k g .70 .4 .136$

Pan, Y., \& Zinkhan, G. M. (2006). Determinants of retail patronage: a meta-analytical perspective. Journal of Retailing, 82(3), 229-243. doi: 10.1016/j.jretai.2005.11.008

Pereira, R. C. F. (2004, setembro). Explorando conceitos e perspectivas da meta-análise em marketing. Anais do Encontro Nacional da Associação Nacional de Pós-Graduação e Pesquisa em Administração, Curitiba, PR, Brasil, 28.

Price, L., \& Arnould, E. J. (1999). Commercial friendships: service provider-client relationships in context. Journal of Marketing, 63(4), 38-56. doi: 10.2307/1251973

Ranaweera, C., \& Prabhu, J. (2003). On the relative importance of customer satisfaction and trust as determinants of customer retention and positive word of mouth. Journal of Targeting, Measurement and Analysis of Marketing, 12(1), 82-90. doi: 10.1057/palgrave.jt.5740100

Reynolds, K. E., \& Arnold, M. J. (2000). Customer loyalty to the salesperson and the store: examining relationship customers in an upscale retail context. The Journal of Personal Selling \& Sales Management, 20(2), 89-98.

Reynolds, K. E., \& Beatty, S. E. (1999). Customer benefits and company consequences of customersalesperson relationships in retailing. Journal of Retailing, 75(1), 11-32. doi: 10.1016/S00224359(99)80002-5

Richins, M. L. (1983). Negative word-of-mouth by dissatisfied consumers: a pilot study. Journal of Marketing, 47(1), 68-78. doi: 10.2307/3203428 
Rosenthal, R. (1979). The 'file drawer problem' and tolerance for null results. Psychological Bulletin, 86(3), 638-641. doi: 10.1037/0033-2909.86.3.638

Samson, A. (2006). Understanding the buzz that matters: negative vs positive word of mouth. International. Journal of Market Research, 48(6), 647-657.

Schoefer, K., \& Diamantopoulos, A. (2008). The role of emotions in translating perceptions of (in)justice into postcomplaint behavioral responses. Journal of Service Research, 11(1), 91-103. doi: $10.1177 / 1094670508319091$

Sirdeshmukh, D., Singh, J., \& Sabol, B. (2002). Consumer trust, value, and loyalty in relational exchanges. Journal of Marketing, 66(1), 15-37. doi: 10.1509/jmkg.66.1.15.18449

Söderlund, M. (1998). Customer satisfaction and its consequences on customer behaviour revisited. International Journal of Service Industry Management, 9(2), 169-188. doi: $10.1108 / 09564239810210532$

Söderlund, M. (2006). Measuring customer loyalty with multi-item scales: a case for caution. International Journal of Service Industry Management, 17(1), 76-98. doi: $10.1108 / 09564230610651598$

Söderlund, M., \& Rosengren, S. (2007). Receiving word-of-mouth from the service customer: an emotion-based effectiveness assessment. Journal of Retailing and Consumer Services, 14(2), 123-136. doi: 10.1016/j.jretconser.2006.10.001

Steiner, P. J., Neto, Schlemer, C. B., \& Pádua, F. P., Jr. (2004, novembro). Um estudo sobre o arrependimento de adolescentes proprietários de aparelho celular na cidade de Curitiba. Anais do Encontro de Marketing da ANPAD, Porto Alegre, RS, Brasil, 1.

Swan, J. E., \& Oliver, R. E. (1989). Postpurchase communications by consumers. Journal of Retailing, 65(4), 516-533.

Sweeney, J. C., Soutar, G. N., \& Mazzarol, T. (2005, dezembro). The difference between positive and negative word-of- mouth-emotion as a differentiator. Anais do ANZMAC Conference: Broadening the Boundaries, Perth, Austrália.

Szymanski, D. M, \& Henard, D. H. (2001). Customer satisfaction: a meta-analysis of the empirical evidence. Journal of the Academy of Marketing Science, 29(1), 16-35. doi: $10.1177 / 009207030102900102$

Vieira, V. A. (2008, maio). The relationship between market orientation and business performance: a Brazilian meta-analysis. Anais do Encontro de Marketing da ANPAD, Curitiba, PR, Brasil, 3.

Wangenheim, F. V. (2005). Postswitching negative word of mouth. Journal of Service Research, 8(1), 67-78. doi: 10.1177/1094670505276684

Wangenheim, F. V., \& Bayón, T. (2007). The chain from customer satisfaction via word-of-mouth referrals to new customer acquisition. Journal of the Academy of Marketing Science, 35(2), 233 249. doi: 10.1007/s11747-007-0037-1

Westbrook, R. A. (1987). Product/consumption based affective responses and postpurchase processes. Journal of Marketing Research, 24(3), 258-270. doi: 10.2307/3151636

Westbrook, R. A., \& Oliver, R. L. (1991). The dimensionality of consumption emotion patterns and consumer satisfaction. Journal of Consumer Research, 18(1), 84-91. doi: 10.1086/209243 
Wolf, F. M. (1986). Meta-analysis: quantitative methods for research synthesis. Beverly Hills, CA: Sage.

Zeithaml, V. A., Berry, L. L., \& Parasuraman, A. (1993). The nature and determinants of customer expectations of service. Journal of the Academy of Marketing Science, 21(1), 1-12. doi: $10.1177 / 0092070393211001$

Zeithaml, V. A., Berry, L. L., \& Parasuraman, A. (1996). The behavioral consequences of service quality. Journal of Marketing, 60(2), 31-47. doi: 10.2307/1251929 